\title{
An Efficient Data Compression Approach based on Entropic Coding for Network Devices with Limited Resources
}

\author{
Elie Tagne Fute, Hugues Marie Kamdjou, Alain Bertrand Bomgni, and Armand Nzeukou
}

\begin{abstract}
The expansion of sensitive data deriving from a variety of applications has required the need to transmit and/or archive them with increased performance in terms of quality, transmission delay or storage volume.

However, lossless compression techniques are almost unacceptable in the application fields where data does not allow alterations because of the fact that loss of crucial information can distort the analysis.

This paper introduces MediCompress, a lightweight lossless data compression approach for irretrievable data like those from the medical or astronomy fields. The proposed approachis based on entropic Arithmetic coding, Run-length encoding, Burrows-wheeler transform and Move-to-front encoding. The results obtained on medical images have an interesting Compression Ratio (CR) in comparison with the lossless compressor SPIHT and a better Peak Signal to Noise Ratio (PSNR) and Mean Squared Error (MSE) than SPIHT and JPEG2000.
\end{abstract}

Index Terms-Big Data; Entropic Coding; Image Compression; Lossless Compression; MSE; PSNR; Telemedicine

\section{INTRODUCTION}

For the World Health Organization specialized group, telematics is defined as a form of cooperative medical practice connecting from a distance a patient and a doctor (or several health professionals) through information communication technology [1]. In some of its applications such as medical imaging, telemonitoring or remote monitoring of a patient, remote control, telemedicine and telesurgery [2]. The amount of digital medical images is steadily increasing, which requires a crucial treatment on the quality and size of the images to be stored because of their nature or because of the context in which the compression takes place (because of regulations or legislations). These methods aim at reducing images to a more compact form at the cost of compression and decompression effort [3].

This paper proposes an efficient lossless data compression

Published on October 05, 2019

Elie Tagne Fute, PhD, Department of Mathematics and Computer Science at the University of Dschang, Cameroon; Department of Computer Engineering at the University of Buea, Cameroon (email:eliefute@yahoo.fr).

Hugues Marie Kamdjou, PhD student, Department of Mathematics and Computer Science at the University of Dschang, Cameroon (e-mail: khuguesmarie@yahoo.com).

Alain Bertrand Bomgni, PhD, Department of Mathematics and Computer Science at the University of Dschang, Cameroon (e-mail: alain.bomgni@gmail.com).

Armand Nzeukou, Professor, Department of IUT-FV at the University of Dschang, Cameroon (e-mail: armand.nzeukou@gmail.com). technique for resource-limited devices. We name this approach as MediCompress and apply to images compression while optimizing the processing time and guaranteeing the integrity of the original image. MediCompress is based on entropic coding and does not allow alterations. The main benefit of this approach is its low complexity. The data is reduced without any quality loss, while reducing the image data size by removing unnecessary meta-data from the submitted files. Nevertheless, the original data can be restored and reconstructed from the compressed file data. Our lossless approach will only temporarily delete the file data, this allows it to be transferred quickly, to optimize loading and processing speeds in memory. While the amount of space that will save is not as much as if we were to use lossy compression, it does give us higher quality images and the option to fully restore.

The remainder of this work is organized as follows: in section 2, we review relevant existing research on data compression; in section 3, we present our approach MediCompress and its main components; in section 4, we present the implementations and interpretation of results. Finally section 5 provides conclusion and future directions.

\section{RELATED REVIEW}

\section{A. Theoretical framework}

Data compression is considered the starting point for Claude Shannon's 1948 publication of "The Mathematical Theory of Communication" [4]. In this theory he particularly introduced the notion of quantity of information $\mathrm{I}(\mathrm{X}=\mathrm{x})$ of an event $\mathrm{X}=\mathrm{x}$, and the entropy $\mathrm{H}(\mathrm{X})$ of a discrete variable which defines the limit of $\mathrm{X}$ compression. Image compression technique basically divided into two parts: lossy technique and lossless technique. Lossless compression algorithm involves no loss of information. If data have been loss-lessly compressed the original data most be recovered exactly from the compressed data. Lossy compression algorithm involves some loss of information and data that have been compressed using lossy technique generally cannot be recovered or reconstructed exactly.

\section{B. Variable length coding, adaptive Huffman coding}

The general idea of this type of code is to assign a shorter code to the most frequent characters, and a longer code to the least frequent characters. Adaptive Huffman encoding requires prior knowledge of the probability of symbols appearing, and then the encoding procedure is as follows: we identify a prefix code from a discrete source $X$ to a 
binary tree with $\|\mathrm{X}\|$ labeled sheets by the letters of $\mathrm{X}$. Suppose we have already read $\mathrm{n}$ characters in the text, corresponding to $\mathrm{K}$ separate letters. We make $\mathrm{X}_{\mathrm{n}}$ a source of $(\mathrm{K}+1)$ letters consisting of $\mathrm{K}$ letters already appeared to which we assign a probability proportional to the number of occurrences, and a $(\mathrm{K}+1)^{\text {th }}$ letter (empty) to which we attribute the probability 0 ; we build a Huffman code $T_{n}$ from this source; the $(n+1)^{\text {th }}$ letter is read and is coded by its codeword if it exists, by the $(\mathrm{K}+1)^{\text {th }}$ codeword followed by the ASCII code otherwise. For this purpose, Huffman uses a tree based on the basic notions of information theory (Shannon-Fano) namely the notion of quantity of information and entropy [5].

\section{Adaptive arithmetic coding}

This encoding replaces the set of symbols read by a single real number which is between $[0 ; 1)$. At the first iteration, the interval is divided into $n$ segments of equal length. Each time a symbol is received; the algorithm calculates the new probability distribution and updates the symbols. On the other hand, if we really consider all the possible sequences, this number can include an infinite number of digits in its binary representation [6].

\section{Run-length encoding}

Its principle is to replace several appearances of the same symbol by a copy of this symbol and the number of times that it appears consecutively in the data [7].

\section{E. Burrows-Wheeler Transform}

Transformed from Burrows-Wheeler's principle is to rearrange a string of characters into a series of similar characters. The result contains exactly the same characters as at the input, the only difference is the order in which they appear [8].

F. Dictionary-based method: PPM Compression, LZMA, LZ77, LZ78, LZO, 7-Zip, WinRar, WinZip, Bzip2 and Gzip

7-Zip is the compression algorithm that integrates the following methods: LZMA (Enhanced and Optimized Version of LZ77), LZMA2 (Enhanced Version of LZMA), PPMD (partial recognition prediction algorithm which consists of to predict the next symbol to be encoded based on the previous $\mathrm{n}$ symbols), BCJ, BCJ2 (Converter for 32bit $\mathrm{x} 86$ executables), Bzip2 (compression using the BWT transform, LZMA and Huffman coding) and Deflate or Gzip (similar to LZO is a standard algorithm based on LZ77 and Huffman). Unlike LZ77, the LZ78 no longer uses the sliding window but a dictionary containing the byte strings already encountered. WinRar uses LZ77 and Huffman while WinZip uses LZ77, LZMA, BWT and Huffman [9].

\section{$G$. The discrete cosine transforms (DCT)}

DCT is a mathematical function used for destructive compression of data, especially for sounds, images and videos according to JPEG and MPEG standards. During the data compression operation in these different formats, the algorithm transforms the pixels of the image or the samples of the audio sequence into frequencies, while eliminating the frequencies that do not correspond to the relevant data for the eye or the human ear [10].

\section{H. Joint Photographic Experts Group (JPEG) compression}

JPEG compression encodes the colors of the image in any format, however the best compression ratios are achieved with luminance/chrominance color coding because the human eye is quite sensitive to the luminance (brightness) but not very sensitive to chrominance (the hue) of an image. In order to exploit this property, the algorithm converts the original image from its initial color model (usually RGB (Red, Green, Blue)) to the YIQ chrominance/luminance type (the $\mathrm{Y}, \mathrm{I}$, and $\mathrm{Q}$ represent luminance, interpolation and quadrature respectively [11].

\section{Fractal compression}

According to fractal compression, any image is a finite set of geometric transformations (rotations, translations, enlargements, reductions) applied to subsets of identical patterns and variable sizes that compose it. This method consists to detect on the one hand the recurrence of the patterns at different scales, and on the other hand to eliminate the redundancy of information in the image [12].

\section{J. Discrete Wavelet Transform (DWT)}

DWT is a method of image compression based on mathematical theory of signal analysis. Wavelets are sets of elementary signals from which a complex signal is reconstructed. The idea of this transform is to determine the correlation of several wavelets (compressed or dilated mother wavelet) with the signal, while highlighting the details and the overall look [13]. DWT is a multiresolution/multi-frequency representation. It is a tool that splits data, functions or operators into frequency components at a scale resolution.

\section{K. Coding of distributed sources}

The coding of distributed sources do not allow communication between encoders (decoding is always done jointly) (see Fig. 1) [14].

\section{Pipelined In-Network Compression}

In this technique, the data collected by the sensor is stored in the buffer of the aggregation node for a certain time lapse [15]. During this time, the data packets are combined into one packet while suppressing the redundancies (see Fig. 2).

\section{Analysis of above methods}

In view of the above techniques, we realize that some are general while others are specialized in data of a certain nature, for example: images, videos, sounds, text. Some perform a lossless encoding and others, with a loss of information. The former are mandated to reconstruct the original data without any difference after they undergo the compression and decompression steps. The latter may alter the data after compression and decompression provided that the reconstructed data is relatively similar to the original data [16].

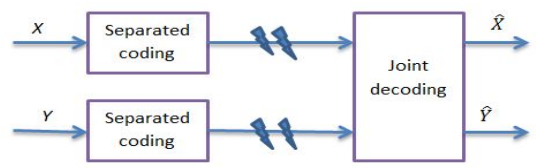

Fig. 1.Example of a Distributed Source Coding System 


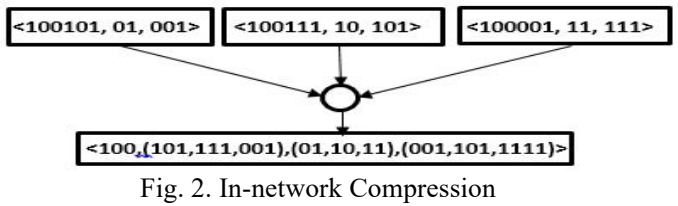

The DCT-based methods, such as the JPEG algorithm, aim to nullify low value coefficients that represent little information in the image, and reduce the dynamics of others. Thus, it introduces "artifacts" ripple due to the truncation of high frequency coefficients; which generates a loss of information. Despite the fact that the DWT is a global transformation, the block effect affects the quality of the image, because of the error due to the quantization of the coefficients. The disadvantage of the dictionary methods is that the size of the dictionary is limited by the available memory [17].

\section{THE PROPOSED APPROACH OF COMPRESSION: MEDICOMPRES}

MediCompress is a lossless compression approach of data that does not allow alterations. Our aims consist to reduce the amount of information represented by a pixel, without losing the originality of the pixel. We begin by splitting an input image into $8 \times 8$ rows and columns, and then compress each block with our algorithm. At final stage sum of all individual compressed bloc which not only provide better result but also the information content will be kept secure.

The architecture (Fig. 3) of this approach is based on the codings: ARIthmetic revised (ARI), Run-Length Encoding revised (RLE), Burrows-Wheeler Transform revised (BWT) and Move-To-Front Encoding revised (MTF). The reason why these codings have been chosen is that they are particularly valuable in constrained resource applications (energy and memory) because their implementations involve simple instructions for adding and offsetting integer values [18].

The encoding inspired by adaptive arithmetic coding consists of replacing the set of symbols read by a single real number that is in the interval $[0,1]$. Either to code $x^{n} \in E^{n}$ to the order $k$ chosen beforehand; we proceed by iteration, for $t \geq 0$, put $x^{t}=x_{I} ; \ldots ; x_{t}$ and suppose treated the first $\mathrm{t}$ symbols, $t \geq 0$; $t=0$ meaning that the coding has not started yet. To treat the $(\mathrm{t}+1)^{\mathrm{th}}$, we update the probabilities of transitions as follows:

$$
\gamma(t)(i \mid j)=\frac{n^{(t)}(i \mid j)+1}{n^{(t)}(j)+m} \quad \text { Withi } \in E ; j \in E^{k} ; n^{(t)}(i \mid j) \text { and }
$$

$n^{(t)}(j)$ which are respective occurrence numbers of $i$ after $j$ and of $j$ in $x^{t} ; n^{(t)}(j)$ not counting an appearance of $j$ at the end of this chain. Putting $j=x_{t-k+1} ; \ldots ; x_{t}$, the current state and we cut the interval according to the probabilities of $\gamma(t)(i \mid j)$, an interval corresponding to a state $i$ of $E$. We choose as the current interval that corresponds to $\mathrm{x}_{\mathrm{t}+1}$. Once the last processed symbol and the noted current interval ([a; $\mathrm{b}[)$, there are two consecutives dyadic numbers $[-\log (b-a)]$ in the current range. We take for code $x^{n}$ the fractional part of the largest of these numbers.

The use of this method of compression has a defect due to the use of a table of fixed statistics translating the same problems as for the Huffman coding. That is to say that the compression of a file with atypical statistics (symbols to be poorly represented are found with a high probability of appearance) will be larger after compression. To overcome this, the most effective solution is to use an adaptive array frequencies have the same law of probability; then at each meeting of a symbol, the statistics and the intervals are adapted (see Algorithm 1 and 2).

In order to increase the chances of having a good compression ratio, we precede this coding of the transformations inspired by Run-Length Encoding, MoveTo-Front and Burrows-Wheeler Transform.

The encoding inspired by Run-Length Encoding consist to replace all sequences of characters or similar bits by a number representing the number of repetitions of the character or bit followed by that character. For example, if we have a series of zero (0) in a document, 00000 will be replaced by a message of type 50 . Therefore, a means is needed to differentiate the message 50 from the sequence 50 in the original data. For this purpose, we use an escape sequence that is to say a new symbol that is introduced into our alphabet. It suffices then to transmit the escape sequence before transmitting the number of times a symbol appears (see Algorithm 3 and 4) [14].

This coding is effective for compressing data where repetitions of consecutive elements are numerous (blackand-white image), which is not always the case; this is why we precede this coding of transformations inspired by Move-To-Front and Burrows-Wheeler Transform; in order to increase the chances of having more characters or repeated bits.

The encoding inspired by Move-To-Front encoding is intended to bring up zero (0) when the input word contains identical character sequences, thus producing highly compressible data (see Algorithm 5 and 6). The transformation of a chain $\mathrm{L}$ of length $\mathrm{N}$ into a vector $\mathrm{R}$ of length $\mathrm{N}$ is as follows:

- The characters of an alphabet A are placed in a list Y which contains only one and only appearance of each character.

- For each $i$, such that $i=0, \ldots, N-1$ :

- $R[i]$ is equal to the number of characters preceding the character $\mathrm{L}[\mathrm{i}]$ in the list $\mathrm{Y}$.

- If the code is the same as that of the last coded character, then they are identical: we return zero (0).

- L[i] is placed at the top of list $\mathrm{Y}$ (the other characters are shifted).

We precede this Burrows-Wheeler Transform-based transform coding, to increase the chances of having more consecutive characters or bits repeated.

The encoding inspired by Burrows-Wheeler's transform coding consists of rearranging a string of characters into sets of similar characters. The result contains exactly the same characters as the input. The only difference is the order in which they appear. This is useful for compression because it tends to be easy to compress a string that has a series of repeated characters. Principles of the algorithm:

- The first step is to read in a block of $\mathrm{N}$ symbols $\mathrm{C}_{0} \ldots \mathrm{C}_{\mathrm{N}-1}$.

- The next step, we consider a block as a cyclic buffer with $\mathrm{N}$ strings (rotations) $\mathrm{S}_{0} \ldots \mathrm{S}_{\mathrm{N}-1}$ which can be constructed so that: $\mathrm{S}_{0}=\mathrm{C}_{0} ; \ldots ; \mathrm{C}_{\mathrm{N}-1} \ldots \quad \mathrm{S}_{\mathrm{N}-1}=\mathrm{C}_{\mathrm{N}-}$ ${ }_{1} ; \mathrm{C}_{0} ; \ldots ; \mathrm{C}_{\mathrm{N}-2}$.

- The third step is to sort lexicographically $\mathrm{S}: \mathrm{S}_{0} \ldots \mathrm{S}_{\mathrm{N}-1}$. 
- The last stage of the transformation is the output of a chain L, composed of the last character of each of the rotations in their sorting order with I the number of the sorted line containing $\mathrm{S}_{0}$

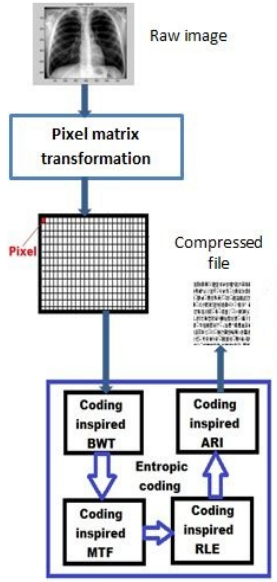

Compression

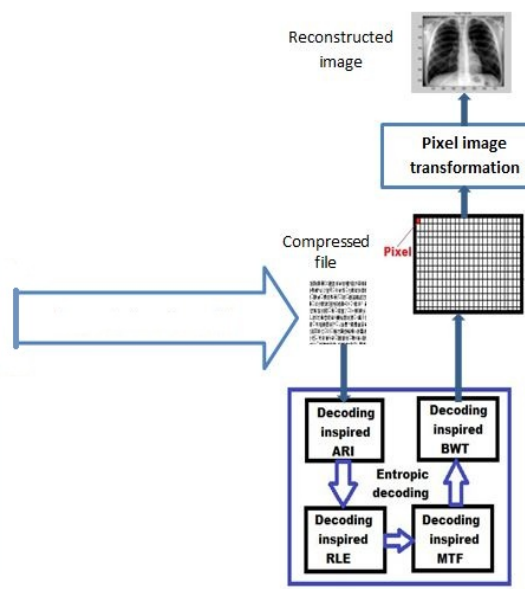

Decompression
Fig. 3.Architecture of our MediCompress approach

Algorithm 1: Component_base_ARI_coding;

1. Input: source file;

2. Output: compressed file;

3. BEGIN

4.low $<-0$; up $<-1$; range $<-1$;

5. WHILE (Not_end_of_file) DO

6. symbol <- get symbol ;

7. low $<-$ low + range * lower of current symbol;

8. up $<-$ low + range * upper_of_current_symbol;

9. range <- up - low;

10.Ouput_a_code_so_that low $\leq$ code $<$ up;

11.ENDwhile

12. END

Algorithm 2: Component_base_ARI_decoding;

1. Input: compresed file;

2. Output: decompressed file;

3. BEGIN

4. low $<-0$; up $<-1$; range $<-1$;

5. WHILE (Not_end of file) DO

6. value <-get code_of_symbol;

7. symbol<-symbol_suc_that_upper_symbol

$\leq(($ value - low $) /($ up - low $))<\bar{l}$ low_symbol ;

8. range <- up - low;

9. up $<-$ low + range $*$ low of current symbol ;

10. low $<-$ low + range * up_of_symbol ;

11. Return symbol;

12. ENDwhile

13. END

Algorithm 3: Component_base_RLE_coding;

1. Input: source file;

2. Output:compresse_file;

3. BEGIN

4. meter $<-0$; boolean $<-$ false ;

5.previous_symbol <- get current_symbol;

6. WHILE ((current_symbol<-next_symbol) $\geq 0)$ DO

7. put current_symbolin_the_buffer;

8. IF (current_symbol==previous_symbol) THEN

9. count $<-0$;

10. boolean $<$-false ;

11. WHILE ((meter <255) and ((current_symbol<- next_symbole $) \geq 0)$ and $($ boolean $==$ false)) $\mathrm{DO}$

12. IF (current_symbol==previous_symbol) THEN

13. meter $<-$ meter +1 ;

14. ELSE

15. boolean $<-$ true;

16.ENDif

17.ENDwhile

18. put meter in_the_buffer;

19. IF ((meter $\neq 255)$ and (current_symbol $\geq 0))$ THEN

20. put symbol in_the_buffer;

21.ENDif

22.ENDif

23. previous_symbol<-current_symbol;

24.ENDwhile

25. END

Algorithm 4: Component_base_RLE_decoding;

1. Input: compresed file;

2. Output: decompressed file;

3. BEGIN

4. meter <- 0; previous_symbol<-current_symbol;

5. WHILE ((current_symbol<- next symbol) $\geq 0)$ DO

6. put symbol in the buffer;

7. IF (current_symbol $==$ previous_symbol) THEN

8. meter<-next_symbol;

9. WHILE ((meter <- meter - 1) >0) DO

10. put current_symbol in_the_buffer;

11. ENDwhile

12.ENDif

13. previous_symbol<-current_symbol;

14.ENDwhile

15. END

Algorithm 5: Component_base_MTF_coding;

1. Input: source file;

2. Output: compressed file;

3. BEGIN

4. Initialize the_symbol_table;

5. WHILE (Not_end of file) DO

6. $S<$-get symbol;

7.put position_S_symbol_of_the_symbol_tablein_the_buffer

8. move the symbol_S_to_the head_of_the_table_symbols;

9.ENDwhile

10. END

Algorithm 6: Component_base_MT_decoding;

1. Input: compresed file;

2. Output: decompressed_file;

3.BEGIN

4. Initialize the_symbol_table;

5. WHILE (Not end of file) DO

6. $\quad P<-$ get symbol_position;

7. put symbol

in_position_P_of_the_symbol_table_in_the_buffer;

8. move the symbol Sto the head of the table symbols;

9.ENDwhile

10. END

\section{IMPLEMENTATIONS AND INTERPRETATION OF RESULTS}

To find the optimal order of our components (BWT, 
MTF, RLE and ARI) namely MediCompress, we compressed the X-ray medical image samples of the tooth, knee, thorax and hand (Fig. 4) [19]. Using all possible cases of the components of our approach (see Table I), although we insist on compression in the description of our experiments, we have always developed the decompress methods to make sure that our experimental results come from valid compression and decompression algorithms implementations.

The results of our experiments obtained on a laptop computer (Pentium 4, HDD: $500 \mathrm{~GB}$, CPU: 2: $5 \mathrm{GHz}$ (4CPUs), RAM: $4 \mathrm{~GB}$ and OS: Ubuntu 12.0432 bits) are presented in Table I, Table II and Table III.

With regard to the plots of the experimental results presented in Fig. 5, we find that the optimal order of the components of our approach is MediCompress, because its red color plot has the best Compression Ratio (CR) defined as:

$C R=\left(1-\left(\frac{\text { image size after encoding }}{\text { original image size }}\right)\right) \times 100 ;$ on the four sample images compared to the other component orders (Fig. 5 and Fig. 6).

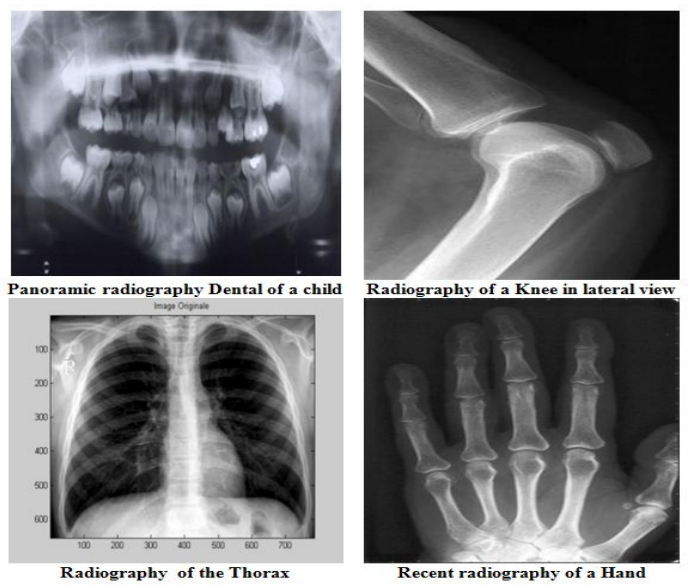

Fig. 4.Overview of sample images

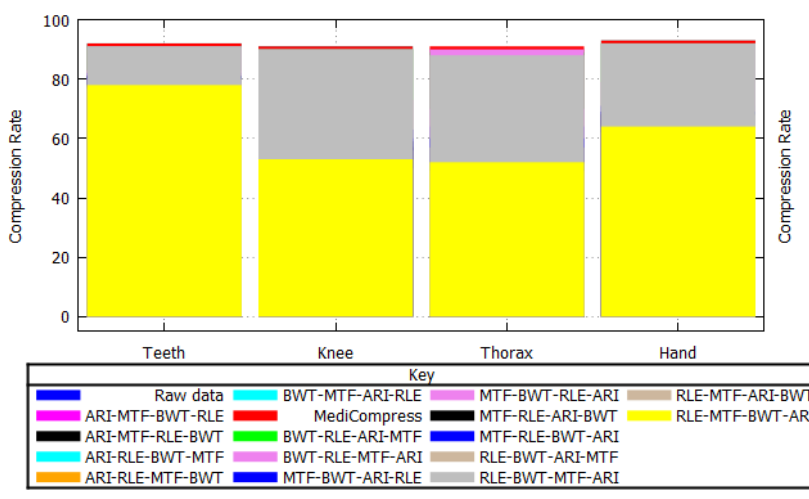

Fig. 5.Histogram of experiments (size in bytes)

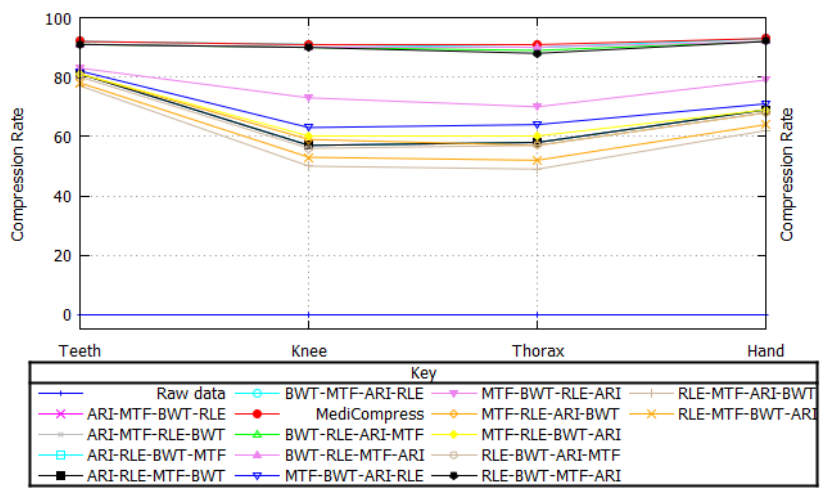

Fig. 6.Results Compression Ratio (\%) of experiments (size in bytes)

\section{A. Comparison and discussion}

To verify the effectiveness of our MediCompress approach, on database images (Fig. 4); we did a comparative study of the results with those obtained from lossless compression approaches in classical networks such as: SPIHT, Arithmetic, Huffman, Run-length encoding, Burrowswheeler transform Move-to-front encoding, Winrar and Winzip; Table II presents the results obtained on four samples of medical images.

The quality of image reconstructed is measure using the Peak Signal to Noise Ratio (PSNR), defined as:

PSNR $($ in $d B)=10 \log _{10}\left(\frac{(P i c)^{2}}{M S E}\right)$; Here, Pic is the maximum possible pixel value of the original image, in this case the pixels are represented using 8 bits per sample, this is 255. The Mean Squared Error (MSE) is defined as:

$M S E=\frac{1}{N M} \sum_{i=1}^{N} \sum_{j=1}^{M}\left(x_{i}-y_{j}\right)^{2}$, with $\mathrm{N}$, M represented respectively the length and width of the image, and $\mathrm{x}_{\mathrm{i}}, \mathrm{y}_{\mathrm{j}}$ represented respectively the value of the original image and reconstructed image.

TABLE I: RESULTS OF EXPERIMENTS (SIZE IN BYTES)

\begin{tabular}{lllll}
\hline Compressor & Teeth & Knee & Thorax & Hand \\
\hline Original file size & 405054 & 256390 & 379369 & 391554 \\
\hline ARI-MTF-BWT-RLE & 74098 & 108030 & 155949 & 119133 \\
\hline ARI-MTF-RLE-BWT & 74113 & 108048 & 155979 & 119107 \\
\hline ARI-RLE-BWT-MTF & 74099 & 108038 & 155988 & 119112 \\
\hline ARI-RLE-MTF-BWT & 74099 & 108037 & 155988 & 119111 \\
\hline BWT-MTF-ARI-RLE & 30196 & 21834 & 35904 & 25303 \\
\hline MediCompress & $\mathbf{2 8 6 0 9}$ & $\mathbf{2 1 2 6 8}$ & $\mathbf{3 3 0 8 9}$ & 23649 \\
\hline BWT-RLE-ARI-MTF & 34536 & 25531 & 38367 & 28120 \\
\hline BWT-RLE-MTF-ARI & 34028 & 24761 & 37738 & 27452 \\
\hline MTF-BWT-ARI-RLE & 70720 & 94016 & 134610 & 110682 \\
\hline MTF-BWT-RLE-ARI & 66387 & 68382 & 113373 & 81264 \\
\hline MTF-RLE-ARI-BWT & 75936 & 103705 & 162111 & 122351 \\
\hline MTF-RLE-BWT-ARI & 74276 & 100375 & 151523 & 118582 \\
\hline RLE-BWT-ARI-MTF & 78316 & 111959 & 162420 & 123552 \\
\hline RLE-BWT-MTF-ARI & 33680 & 25215 & 42599 & 29855 \\
\hline RLE-MTF-ARI-BWT & 89871 & 127460 & 191967 & 146649 \\
\hline RLE-MTF-BWT-ARI & 86618 & 119482 & 178546 & 138232 \\
\hline
\end{tabular}

TABLE II: RESULTS OF EXPERIMENTS 2 (SIZE IN BYTES)

\begin{tabular}{lllll}
\hline Compressor & Teeth & Knee & Thorax & Hand \\
\hline Original file size & 405054 & 256390 & 379369 & 391554 \\
\hline MediCompress & 28609 & 21268 & 33089 & 23649 \\
\hline SPIHT & 28341 & 21268 & 31762 & 23529 \\
\hline
\end{tabular}




\begin{tabular}{lllll}
\hline Winrar & 65536 & 36864 & 81920 & 90112 \\
\hline Winzip & 163840 & 49152 & 98304 & 102400 \\
\hline Arithmetic & 77824 & 110592 & 155648 & 118784 \\
\hline Huffman & 77824 & 110592 & 159744 & 122880 \\
\hline Run-length encoding & 204800 & 299008 & 446464 & 335872 \\
\hline Move-to-front & 176128 & 258048 & 380928 & 282624 \\
\hline $\begin{array}{l}\text { Burrows-wheeler } \\
\text { transform }\end{array}$ & 176128 & 258048 & 380928 & 282624 \\
\hline
\end{tabular}

TABLE III: MEAN PSNR (DB) AND MSE COMPARISON BETWEEN JPEG AND PNG AT 0.1 BITS/VOXEL (BPV) ON THE 4 DATABASE IMAGES

\begin{tabular}{lllllllll}
\hline & Teeth & & Knee & & Thorax & Hand & \\
\hline & PSNR & MSE & PSNR & MSE & PSNR & MSE & PSNR & MSE \\
\hline MediCompress & $\mathbf{4 9 , 0 4}$ & $\mathbf{0 . 8 1}$ & $\mathbf{4 9 . 6 1}$ & $\mathbf{0 . 7 1}$ & $\mathbf{5 5 . 3 4}$ & $\mathbf{0 . 1 9}$ & $\mathbf{5 2 . 0 0}$ & $\mathbf{0 . 4 1}$ \\
\hline JPEG & 38.73 & 8.7 & 40.75 & 5.47 & 42.13 & 3.98 & 41.49 & 4.61 \\
\hline SPHIT & 61.68 & 0.21 & 50.20 & 0.62 & 50.57 & 0.57 & 49.32 & 0.76 \\
\hline
\end{tabular}

Based on the plots of the experimental results presented in Fig. 7 and Fig. 8, we find that our approach (MediCompress) whose curve is in red color has a good Compression Ratio compared to the curves of approaches: SPIHT, Arithmetic, Huffman, Run-length encoding, Burrows-wheeler transform Move-to-front encoding, Winrar and Winzip. Table III, Fig. 9 and Fig. 10 show that the MediCompress PSNR (dB) and MSE results have been satisfactory compared to the JPEG2000 standard implemented in OpenJPEG library, and the compressor without loss of the algorithm SPIHT implemented in QccPackSPIHT4 library.

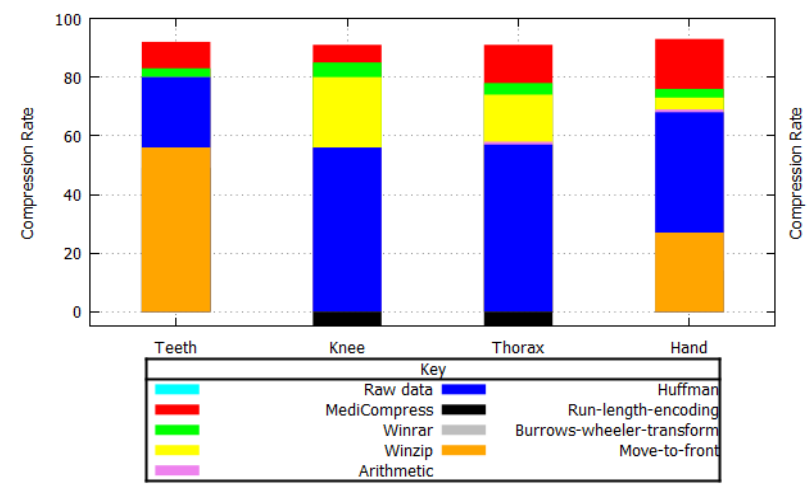

Fig. 7.Comparative histogram Compression Ratio (\%) of MediCompress with other compressions approaches

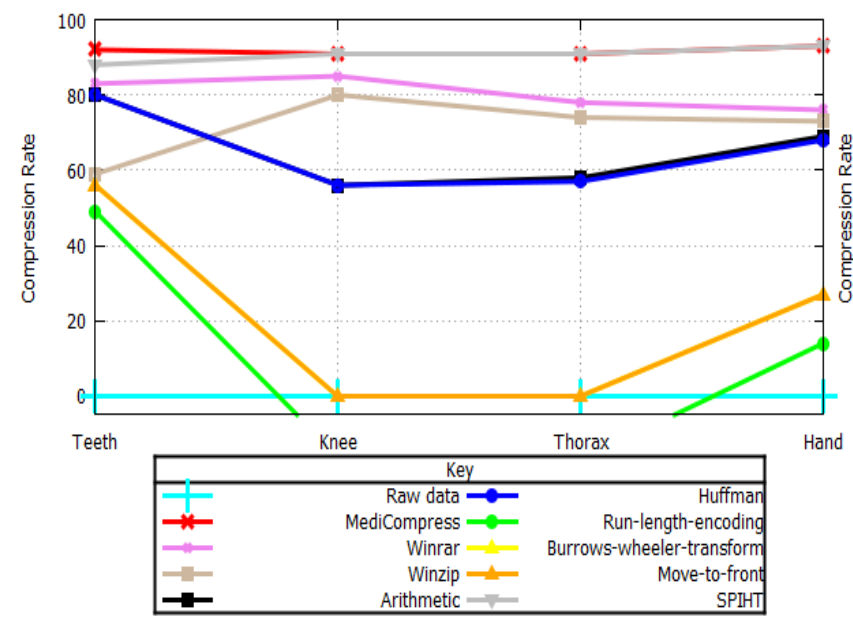

Fig. 8.Comparative Compression Ratio (\%) plots of our approach with other compressions approaches

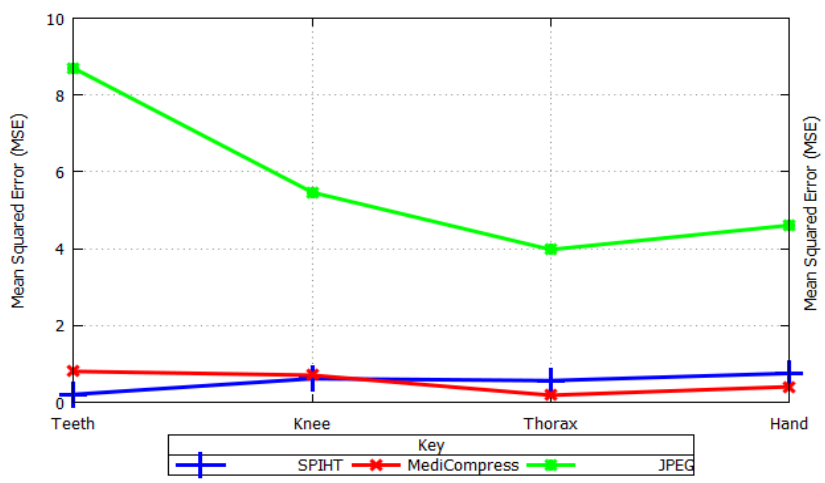

Fig. 9.Comparative Mean Squared Error plots of our approach with other compressions approaches

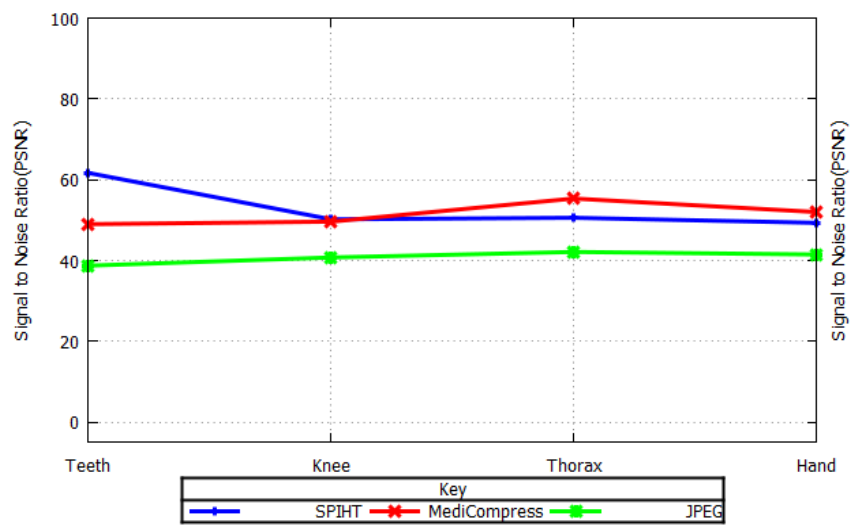

Fig. 10.Comparative Peak Signal to Noise Ratio $(\mathrm{pB})$ plots of our approach with other compressions approaches

\section{CONCLUSION}

In this paper, we have proposed MediCompress: a lossless data compression approach using entropic coding, applied to incorruptible data (like those from the medical field, astronomy field, etc.). This approach is based on classicals compressions techniques namely Arithmetic, Runlength encoding, Burrows-wheeler transform and Move-tofront encoding. In view of the experimental results $(\mathrm{CR}$, MSE and PSNR), we can say that the results presented throughout this work are satisfactory since MediCompress approach effectively reduced the amount of data contained in medical images, and the reconstructed images without loss of information because of security and legislation purpose for the image

\section{ACKNOWLEDGMENT}

This research was supported in part by the Directorate of Central Africa and Great Lakes Region (DCAGLR) of Francophonie University Association (AUF).

\section{REFERENCES}

[1] Victor Shnayder, Borrong Chen, KonradLorincz, Thaddeus R. F. Fulford-Jones and Matt Welsh. Sensor networks for medical care. Division of Engineering and Applied Sciences Harvard University, 2005

[2] O. Chipara, C. Brooks, S. Bhattacharya, C. Lu, R.D. Chamberlain, G.C. Roman, and T.C. Bailey. Reliable Real-time Clinical Monitoring Using Sensor Network Technology. In American Medical Informatics Association Annual Symposium (AMIA), 2009.

[3] Elie T. FUTE, Alain B. BOMGNI and Hugues M. KAMDJOU. An approach to data compression and aggregation in wireless sensor 
networks. International Journal of Computer Science and Telecommunications (IJCST), 2016.

[4] E. Shannon Claude. A mathematical theory of communication. Bell System Tech., pages 379-423, 1948.

[5] Subhra J. Sarkar, Nabendu Kumar Sarkar and IpsitaMondal. Adaptive huffman coding-based approach to reduce the size of power system monitoring parameters. Proceedings of the 5th International Conference on Frontiers in Intelligent Computing, 2017.

[6] Jake McMullen, Boglarka B., MinnaM.Hannula-S., Koen V., Gabriela R.-A., Nonmanut P. and Erno L. Adaptive number knowledge and its relation to arithmetic and pre-algebra knowledge. Journal of the European Association for Research on Learning and Instruction (EARLI), 2017.

[7] Chin-Chen Changa, Chih-Yang Linb and Yu-ZhengWangb. New image steganographic methods using run-length approach. Department of Information Engineering and Computer Science, Feng Chia University,pages 3393-3408, 2006.

[8] Krintz C. Adaptive on-the-fly compression. IEEE Transactions, Parallel and Distributed Systems, pages 15-24, 2006.

[9] Jacob Ziv and Abraham Lempel. Compression of individual sequences via variable-rate coding. IEEE Transactions on Information Theory, pages 530-536, 1978.

[10] Min Li, Biswas Mainak, Kumar Sanjeev and Nguyen Truong. Dctbased phase correlation motion estimation. IEEE, Piscataway NJ, Etats-Unis, 2004.

[11] Pratibha, Sandeep Vijay and Sandeep Kumar Dubey. A Review of Image Transmission using Real Time Technique over WMSN. International Journal of Applied Engineering Research, 2018.

[12] Shikang Kong, Lijuan Sun, Chong Han and JianGuo. An image compression scheme in wireless multimedia sensor networks based on nmf. www.mdpi.com/journal/information, 2017.

[13] Christophe R., Bertrand B., Olivier C., Jean-Michel G., Benoit D. and Jean-Paul Y. Wavelet compression of integral formulations on gpgpu architecture. European Conference on Numerical Methods in Electromagnetism, 2012.

[14] D. Slepian and J. Wolf. Noiseless coding of correlated information sources. IEEE Transactions on Information Theory, pages 471-480, 1973.

[15] Stuti A., Dinesh G., Amitkant P. and Rakesh B. An Extensive Survey on Compression Algorithm for Effective Image Compression. 3rd International Conference on Internet of Things and Connected Technologies, 2018.

[16] Naoto Kimura and ShahramLatifi. A survey on data compression in wireless sensor networks. ITCC'05-International Conference on Information Technology: Coding and Computing, 2005.

[17] R. Das. Performance and power optimization through data compression in network-on-chip architectures. IEEE, High Performance Computer Architecture, pages 215-225, 2008.

[18] Jeong Gil Ko, Chenyang Lu, Mani B. Srivastava, John A. Stankovic, Andreas Terzis and Matt Welsh. Wireless sensor networks for healthcare. School of Engineering and Applied Sciences, Harvard University, 2011

[19] DICOM Viewer to view medical data, https://www.visus.com/en/downloads/jivex-dicomviewer.html.Visited: 2018-20-11.

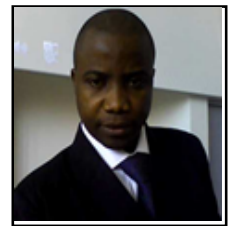

Elie T. FUTE is currently the Head of Department of Computer Engineering, Faculty of Engineering and Technology, University of Buea, Cameroon. He is a lecturer in the Department of Mathematics and Computer Science, Faculty of Science, University of Dschang, Cameroon.

His research works are focused on sensor networks \& applications, multi-agent patrolling, multi-sensor patrolling, computer vision $\&$ applications and data security approaches. From his works has risen several scientific papers published in well known international journals and publications in international conferences with reading panel.

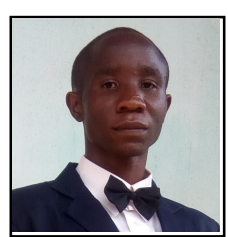

Hugues Marie KAMDJOU is currently a PhD student at Dschang School of Science and Technology (DSST), University of Dschang, Cameroon. Born in Dschang, Cameroon, He holds a Master degree in Mathematics and Computer Science, major on Networks and Distributed Services, domain of Science and Technology,Faculty of Science, University of Dschang, Cameroon in 2016.

His research works are focused on Data Compression, Multimedia Wireless Sensor Networks. Part-time monitor in the department of Mathematics and Computer Science in the University of Dschang, Cameroon. Member in the Doctoral College of Central Africa and the Great Lakes (ACGL) "MathInBio" of the Francophonie University Association (AUF).

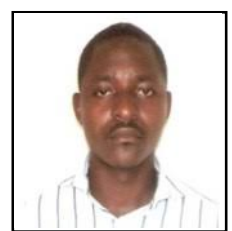

Alain Bertrand BOMGNI is currently a lecturer in the Department of Mathematics and Computer Science, Faculty of Science, University of Dschang, Cameroon. He holds a Ph.D. in Computer Science at the university of Picardie Jules, Amiens, France.

His research works are focused on Distributed Computing, Computer Security and Reliability, Wireless Sensor Network, Interconnection Networks, Parallel algorithms and Architectures.

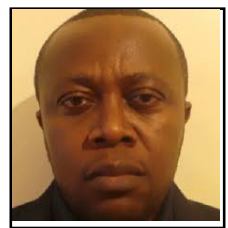

Armand NZEUKOU is a Professor at the University of Dschang, Cameroon. He is currently a lecturer at the Institute of Technology FOTSO Victor of Bandjoun, University of Dschang, Cameroon.

His research works are focused on Remote Sensing, Hydrology, Environmental Impact Assessment, Engineering Environmental Physics. 